\title{
Processing of visual illusions in the frequency and spatial domains
}

\author{
MICHAEL F. BECKER \\ Department of Electrical Engineering, University of Texas at Austin, Austin, Texas 78712
}

and

\author{
JEROME KNOPP \\ M.I.T. Lincoln Laboratory, Lexington, Massachusetts 02173
}

\begin{abstract}
In the results we report here, complex periodic and aperiodic illusions are revealed by filtering with filters containing one or more straight edges. Positive definite filters are used both in the frequency domain and in the space domain to reveal each illusion. Rotating slit and half-plane filters are used in the frequency domain, while processing is done in the spatial domain by convolution with a rotating slit. These two schemes show similar results in revealing the illusory patterns. The implications for human visual processing are discussed.
\end{abstract}

Several investigators have reported successful filtering of figures to reveal patterns which a human viewer perceives as optical illusions. Ginsburg (1971, 1973) found that geometric illustions such as the Mueller-Lyer and Hering illusions could be explained by blurring the object with various low-pass filters in the frequency domain. Kelly (1976) and Kelly and Magnuski (1975) reported that the intensity of the two-dimensional Fourier components govern the visual threshold for several patterns, and that, for the checkerboard, these components are responsible for the illusion of diagonal bars at low-contrast levels.

A filtering interpretation of the Kanizsa triangle (Kanizsa, 1976), or subjective contour illusion, has been attempted by Ginsburg (1975); however, we maintain, as does Tyler (1977), that his arguments are inconclusive. Ginsburg reports that in one case the illusory triangle was revealed by simple low-pass filtering. Analytically, it is impossible for a positive, real, low-pass filter to process a positive real figure and give a result containing sharp edges or lines. High-frequency components are necessary to form sharp edges. In effect, spurious high frequencies are being reinserted into the resulting image by digital processing artifacts. Thus, it is impossible to reveal the illusory triangle with sharp boundaries by using low-pass filtering alone.

Ginsburg also reports that the illusory triangle is revealed by a frequency domain product filter composed of a low-pass filter and a filter representing

The authors would like to express their thanks to Dr. M. T. Manry and Professor Wilson Geisler for many helpful discussions throughout the course of this work and to Dr. D. H. Kelly for a helpful conversation during the formative stages of these experiments. the human visual response function. Since the same digital processing technique is used, this result is also cluttered by digital artifacts. However, since the human response function used by Ginsburg suppresses the zero-frequency component to some extent, the filter produces some "negative" amplitude regions or regions where the light amplitude is phase shifted by $180^{\circ}$. Sharp lines, due to rectification in the detection process, are now possible and will be located at the zero crossings. This occurs because the detection process utilizes the intensity of the light, which is the magnitude squared of the light amplitude. Whether this combination filter can reveal the triangle illusion is still unclear. Ginsburg asserts that since the filtered illusion correlates with a triangle, the illusion has been revealed. Unfortunately, the original figure will also correlate with a triangle. Unless the correlation is shown to significantly increase after filtering, or unless another criterion for illusion explanation is given, this argument is incomplete.

Before presenting our results we will define the operational process by which the revelation of the illusion is to be judged. A correlation between the filtered image and the hypothesized illusory figure is a necessary, but not a sufficient, criteria to make this determination. A higher level of comparison is required, and this is most easily done by visual inspection. Care is crucial at this point, as Tyler (1977) points out, because the human visual system is the same processing system which produces the illusion. This problem may be circumvented by a drastic change in scale. The filtered image is examined under magnification, a part at a time, in order to discover sharp continuous edges where the illusion is to be revealed. If the hypothesized lines or shapes are dis- 


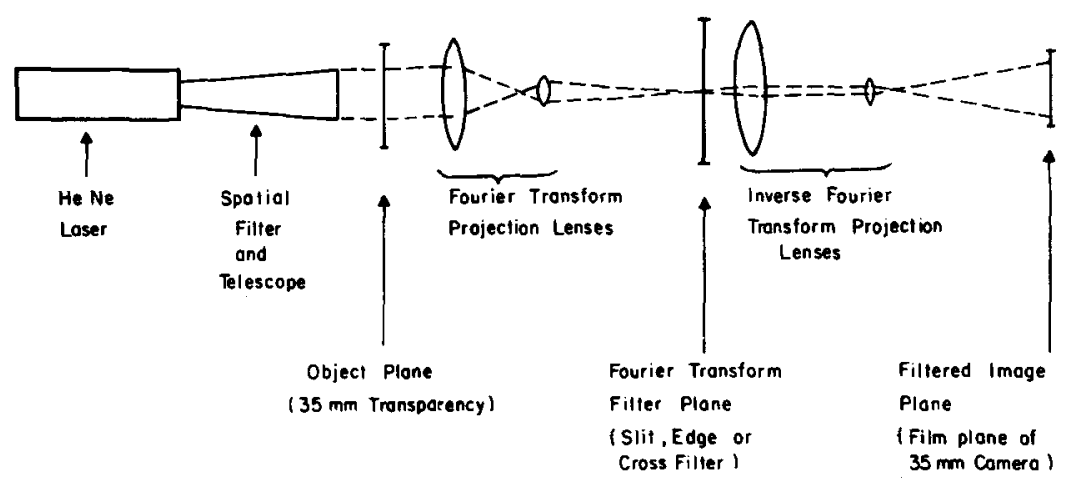

Figure 1. The coherent, frequency domain filtering system.

covered in the filtered image, the illusion is said to be revealed by the filtering process.

The new results we report here reveal more complex periodic and aperiodic illusions by filtering with filters containing one or more straight edges. We obtained our results using two different types of optical computer, one operating in the spatial domain using incoherent light (a convolver) and the other in the Fourier frequency domain using coherent light. We note now and will discuss later the fact that, in terms of input-output relationships, these two methods of processing are similar.

The discovery of one or more filtering systems which reveal illusions does not explain the physiological operation of the human visual system. However, these systems do represent hypothetical models which may now be tested physiologically and experimentally. This testing may, in turn, suggest new optical image-processing systems.

\section{FREQUENCY DOMAIN FILTERING}

Processing of the illusions in the frequency domain was accomplished using the filtering system illustrated in Figure 1. ${ }^{1}$ The coherent beam from a helium-neon laser is spatially filtered, expanded, and collimated using a telescope. The collimated beam illuminates the test subject on a $35-\mathrm{mm}$ transparency in the object plane. ${ }^{2}$ The Fourier transform of the electric (or magnetic) field amplitude transmittance of the transparency is produced at the filter plane. Because each spatial frequency component contained in the input transparency is brought to focus at a separate point in the Fourier transform or filter plane, each may be individually blocked or filtered by placing appropriate masks at this point. In the two-lens transform system, the smaller of the lenses is a projection lens that produces a magnified transform at the filter plane. The magnified transform is easier to view and allows the use of large, easily manipulated filters. By using a projection lens, a spherical phase factor is added to the transform, but this was found to have no noticeable effect on the results as this information is lost in the square law detection process. (This entire arrangement could be replaced with a single long-focal-length lens, but would require an unwieldy bench arrangement.) A second pair of lenses is used in a similar manner to obtain a second Fourier transform and recover a filtered image of reasonable size. This second transform is identical to an inverse transform, except for another phase factor. The filters were inserted in the Fourier transform plane in rotary mounts. The reconstructed, filtered image could then be observed and photographed for single or multiple filter positions. It should be pointed out that although it is the field amplitude that is Fourier transformed, the nonlinear photographic detection process always involves recovery of the field amplitude magnitude squared of the processed image.

The significance of diagonal Fourier components in the checkerboard, Figure $2 b$, to visual thresholds was discovered by Kelly (1976) and Kelly and Magnuski (1975). These components, shown in the magnitude squared of the Fourier transform, Figure 2a, also produce the illusion of diagonal bands at $45^{\circ}$ across the pattern. When a filter in the form of a narrow slit, sufficiently wide to pass only one row of frequency components, is placed at an angle of $45^{\circ}$ in the frequency domain centered on the dc spot, the diagonal light and dark bands are revealed, as shown in Figure 2c. These bands correspond in location to the perceived bands, as shown in Figure 2d, which is an overlay of the filtered and unfiltered images.

A grid pattern, Figure $3 b$, gives a more subtle illusion of light, diagonal lines, intersecting at the common intersections of the dark lines (Schachar, 1976). This report has generated controversy recently because of its comments on the off-diagonal Fourier transform components of the grid pattern (Boulter, 1977; Ginsburg \& Campbell, 1977; Rudee, 1977; Schachar, Black, Hartfield, \& Goldberg, 1977). This illusion may possibly be interpreted as arising from diagonal components of the Fourier transform, shown in the magnitude square transform of Figure 3a. 


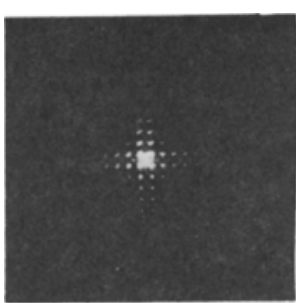

(a)

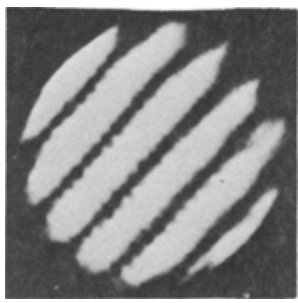

(c)

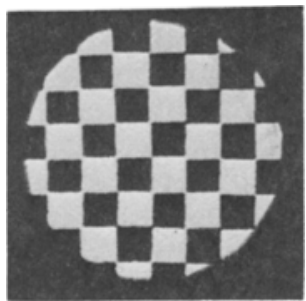

(b)

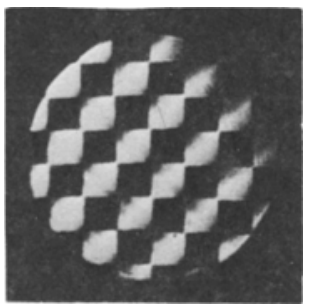

(d)
Figure 2. Results of frequency domain processing of the checkboard. (a) The magnitude squared of the Fourier transform of the checkerboard. (b) The unfiltered checkerboard. (c) Filtered image with slit filter. (d) Overlay of the original image and the filtered image showing correspondence of features.

When the same slit filter is placed at an angle of $45^{\circ}$, selecting a diagonal row of Fourier components, it brings out the sharp diagonal lines as shown in Figure $3 \mathrm{c}$, where the lines run from upper right to lower left. Alternately, a cross-shaped filter selecting both $\pm 45^{\circ}$ diagonals or successive filtering at $\pm 45^{\circ}$ with the slit, reconstructs the entire illusion, as in Figure 3d.

A modification of the slit filter is the half-plane filter. It consists of a straight boundary that is opaque on one side and totally transmitting on the other side. When the edge is positioned to block slightly less than half of the frequency plane, it is, in essence, the sum of a slit plus one-half of all the frequencies present. ${ }^{3}$ This system permits viewing of the original object while simultaneously enhancing the illusion. Examples of half-plane filtering of the grid and checkerboard patterns are shown in Figures $4 a$ and $4 b$, respectively. In both cases, the edge was set at an angle of $45^{\circ}$ in order to bring out one set of illusory bands (from upper right to lower left in these cases). The power of this technique derives from the fact that although the reconstructed illusion is less visible, the original image is transmitted as well. The locations of the various features may be easily compared.

The half-plane filtering technique was also tested with the aperiodic subjective triangle illusion, Figure 5a. The sharp boundaries of the illusion may be made real by filtering successively at $120^{\circ}$ intervals with the edge filter, where the filter is indexed perpendicular to each side of the illusory triangle. The reconstructed image, shown in Figure $5 b$, is a triple exposure of the three filter positions.

\section{SPATIAL DOMAIN PROCESSING}

The process of spatial domain filtering may be described analytically as the two-dimensional convolution $^{4}$ between the original transparency and the filtering function. According to systems theory, a convolution filtering process is identical to a Fourier domain filtering process if the filters are a Fourier transform pair. ${ }^{5}$ If one were to compute the Fourier transform of the slit filter used previously, the result would be the (sine $\mathrm{x}$ ) $/ \mathrm{x}$ function. This is a particularly difficult filter to construct physically because of its negative values. As an approximate alternative, we chose a narrow slit convolution filter, oriented $90^{\circ}$ to the analogous Fourier domain filter. These two systems are only approximately equivalent; however, the approximation is best for the long, narrow slits that we have chosen.

The operation of the narrow slit filter may also be interpreted nonmathematically as a type of pinhole lens. In the narrow dimension of the slit, it behaves exactly as a pinhole lens. It brings all features of the object parallel to the short dimension of the slit into sharp focus at any plane behind the filter. Features parallel to the slit remain blurred or unfocused because many ray paths exist between object and image planes through the long dimension of the slit. Thus, the slit behaves as a directionally selective blurring filter, focusing features normal to, and smearing features parallel to, the slit's long axis.

Two-dimensional optical convolution is easily realized using an incoherent optical computer (Kelly,

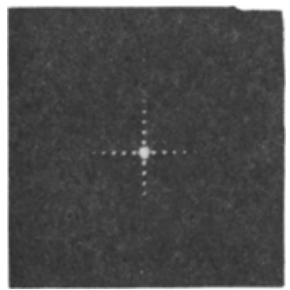

(a)

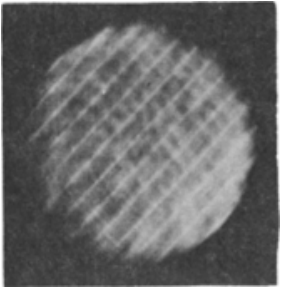

(c)

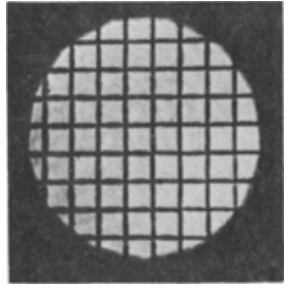

(b)

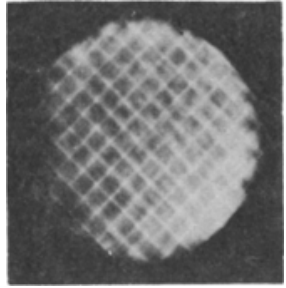

(d)
Figure 3. Results of frequency domain processing of the grid. (a) The magnitude squared of the Fourier transform of the grid. (b) The unfiltered grid. (c) Filtered image with a slit filter. (d) Filtered image with a crossed-slit filter revealing the complete illusion. 


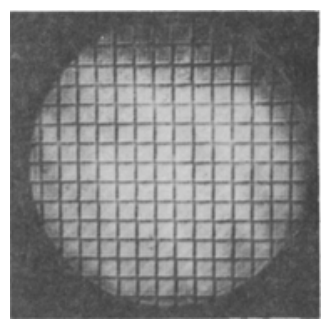

(a)

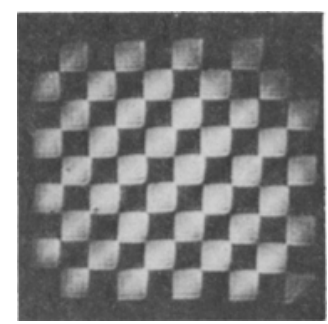

(b)
Figure 4. Results of frequency domain processing with a halfplane filter for (a) the grid, and (b) the checkerboard. The revealed lines go from upper right to lower left in both cases.

1961; Knopp \& Becker, 1978; Trabka \& Roetling, 1964). The optical convolver we used is shown in Figure 6 . In this case, the filter is a narrow, rotatable slit centered on the system's optical axis. The grid and checkerboard objects described previously were processed using this convolution filter, and the results are shown in Figures $7 \mathrm{a}$ and $7 \mathrm{~b}$, respectively. Again, the illusory patterns are elicited in the processed image. For these figures, the slit is parallel to the observed illusion and $45^{\circ}$ to the rectilinear axis of the objects. We also processed the aperiodic, subjective triangle contour by this system. The edges of the illusory triangle are revealed as shown in Figure 5c. In this case, much of the detail of the original object is lost, but the subjective triangle is made real. Here the slit was rotated to three locations $120^{\circ}$ apart, each parallel to a side of the subjective triangle, and a triple exposure was made.

\section{DISCUSSION AND CONCLUSIONS}

We have found that filtering with a slit filter or a half-plane filter in either the frequency domain or in the spatial domain is a powerful technique for revealing optical illusions. These techniques successfully revealed the illusion of diagonal lines or bars in grids and checkerboards, and the triangle in the subjective contour illusion of Kanizsa. Clearly, the periodicity, or lack thereof, in the object has no bearing on the success of this method.

In the case of the subjective contour illusion, the slit and half-plane filters both include high and low frequencies. The high frequencies are necessary to form the sharp edge of the triangle, while the low frequencies are required to make it have a uniform intensity over its wider dimensions. The required presence of both frequency components confirms Tyler's (1977) assertion concerning the necessity of the high-frequency spatial components in revealing this illusion.

In drawing conclusions about the eye-brian system based on data of this type, one must exercise extreme care. Although we have shown two different types of filtering systems which are similar analytically and which give comparable results in revealing illusions, one cannot infer from our results which, if either, model is correct. This data is useful, however, in suggesting possible models for the human visual system which may, in turn, be verified by physiological experiments. Questions of this type have been the subject of investigation for some time. There is an extensive literature suggesting Fourier-type processing

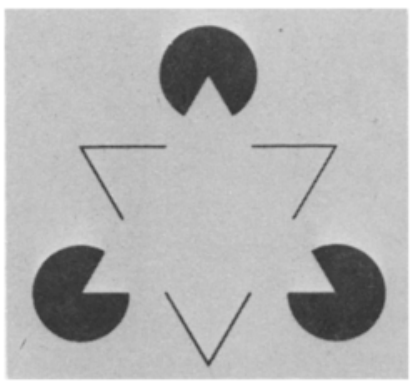

(a)

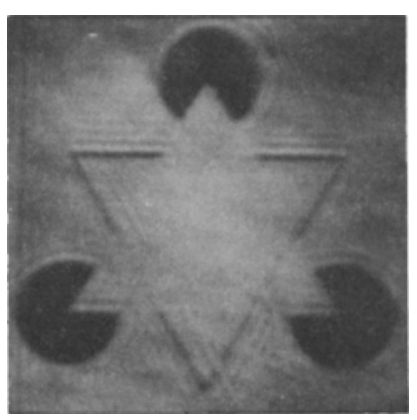

(b)

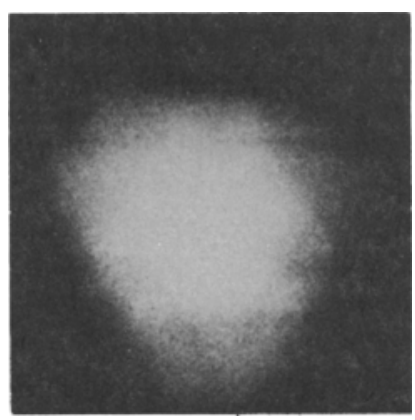

(c)

Figure 5. Processing of the subjective triangle contour illusion. (a) Original object. (b) Filtering in the frequency domain with an edge filter at three successive positions $120^{\circ}$ apart. (c) Filtering in the spatial domain with a slit filter at three successive positions $120^{\circ}$ apart. 


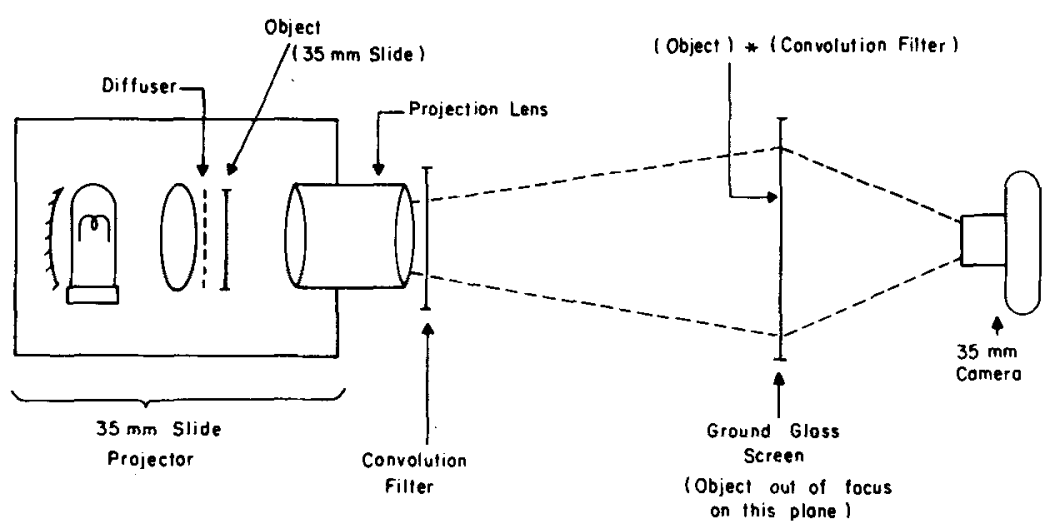

Figure 6. The incoherent, spatial domain filtering system.

in the eye-brain system which is best summarized in a review by Campbell (1974). Other work has emphasized spatially convolving receptive fields which function as separate detection mechanisms for edges, lines, and sinusoidal gratings (Kulikowski \& KingSmith, 1973). However, as we point out here, no strong conclusions about the actual processing mechanism may be drawn from the input-output relationship alone, even when a simple filter description is possible in either domain. Work supporting this thesis has been recently reviewed by Kelly (1977).

Another question which might be raised at this point is, which type of processing system is most simple to implement. The field of picture processing has answered this question for machines. In fact, for optical computers and digital computers, the implementation of spatial domain or convolution processing is simpler than Fourier processing. Only addition and multiplication operations of real positive numbers are required; and the objects, filters, and outputs are positive real functions. On the other hand, Fourier domain processing requires addition, multiplication, and subtraction of complex numbers. The complex numbers or phase information is necessary even when processing with positive real objects and filters. For this reason, Fourier-type optical computing must be done in coherent light while convolution processing may be done in incoherent

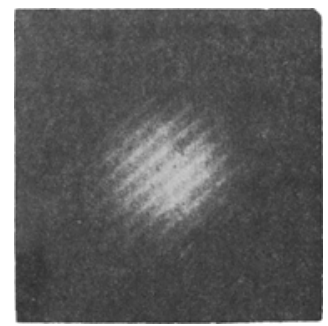

(a)

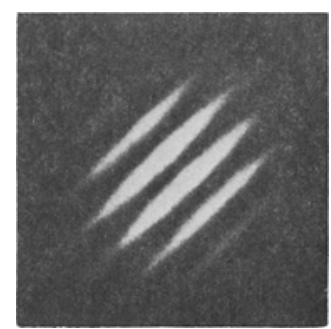

(b)
Figure 7. Results of spatial domain processing with a slit filter for (a) the grid, and (b) the checkerboard. light, as phase information is not important. While Fourier processing is conceptually elegant, convolution processing is more easily implemented in optical and digital computers. We have no evidence that the above is true of the eye-brain system; however, these comparisons with physical systems may point the way for future experiments and models.

\section{REFERENCES}

Boulter, J. F. Technical comment. Science, 1977, 198, 960-961. CAMPBell, F. W. Transmission of spatial information through the visual system. Neurosciences Third Study Program, 1974, 95-103.

Ginsburg, A. P. Psychological correlates of a model of the human visual system. Proceedings: 1971 National Aerospace Electronics Conference (NAECON), Dayton, Ohio, 1971, 283-290.

Ginsburg, A. P. Pattern recognition techniques suggested from psychological correlates of a model of the human visual system. Proceedings: 1973 National Aerospace Electronics Conference (NAECON), Dayton, Ohio, 1973, 309-316.

Ginsburg, A. P. Is the illusory triangle real or imaginary? Nature, 1975, 257, 210-220.

Ginsburg, A. P., \& Campbell, F. W. Technical comment. Science, 1977, 198, 961-962.

Kanizsa, G. Subjective contours. Scientific American, April 1976, 234, 48-52.

KELLY, D. H. Image processing experiment. Journal of the Optical Society of America, 1961, 51, 1095-1101.

Kelly, D. H. Pattern detection and the two-dimensional Fourier transform: Flickering checkerboards and chromatic mechanisms. Vision Research, 1976, 16, 277-287.

Kelly, D. H. Visual contrast sensitivity. Optica Acta, 1977, 24, 107.129 .

Kelly, D. H., \& Magnuski, H. S. Pattern detection and the two-dimensional Fourier transform: Circular targets. Vision Research, 1975, 15, 911-915.

KNopp, J., \& BECKER, M. F. Generalized model for incoherent optical convolvers and correlators. Applied Optics, April 1, 1978, 17, 984-985.

KulikowsKI, J. J., \& KING-SMITH, P. E. Spatial arrangement of line, edge and grating detectors revealed by subthreshold summation. Vision Research, 1973, 13, 1455-1478.

RudeE, M. L. Technical comment. Science, 1977, 198, 960.

SChaChar, R. A. The 'pincushion grid' illusion. Science, 1976, 192, 389.390.

Schachar, R. A., Black, T. D., Hartfield, K. L., \& Goldberg, I. S. Technical comment. Science, 1977, 198, 962. 
Trabka, E. A., \& Roetring, P. G. Image transformations for pattern recognition using incoherent illumination and bipolar aperture masks. Joumal of the Optical Society of America, $1964,54,1242$.

TYLER, C. W. Is the illusory triangle physical or imaginary? Perception, 1977, 6, 603-604.

\section{NOTES}

1. A basic description of frequency domain processors may be found in an introductory optics text, such as F. A. Jenkens and H. E. White, Fundamentals of Optics 4th edition (McGraw-Hill, New York, 1976). A more detailed treatment is given in $J$. Goodman, Fourier Optics (McGraw-Hill, New York, 1968).

2. Because black and white (binary) transparencies were used, the field amplitude and intensity transmittances are the same. For gray scaled transparencies, special care must be taken to adjust the intensity gray scale to a field-amplitude gray scale.

3. This resembles a Schlieren system which blocks slightly more than half the frequency plane, including the dc spot.

4. Two-dimensional convolution is defined as

$f(x, y) * g(x, y)=\int_{-\infty}^{\infty} \int_{-\infty}^{\infty} f\left(x^{\prime}, g^{\prime}\right) g\left(x-x^{\prime}, y-y^{\prime}\right) d x^{\prime} d y^{\prime}$.

5. A summary of two-dimensional systems theory as it applies to optical processing may be found in Goodman (1968) referenced in Note 1 above.

(Received for publication December 20, 1977; revision accepted April 3, 1978.) 